\title{
Analysis of Designation and Symbolic Meanings of Floral Emblems in South Korea as Elements of Garden Tourism and Design
}

\author{
Inhea Kim ${ }^{1,4}$, Jin-Sil Park ${ }^{2}$, and Kyoung-Ok Choi ${ }^{3,4^{*}}$ \\ ${ }^{1}$ Lecturer, Department of Horticulture, Gyeongnam National University of Science and Technology, Jinju 52725, Korea \\ ${ }^{2}$ Master program in Horticulture, Gyeongnam National University of Science and Technology, Jinju 52725, Korea \\ ${ }^{3}$ Professor, Department of Horticulture, Gyeongnam National University of Science and Technology, Jinju 52725, Korea \\ ${ }^{4}$ International Garden Institute, Gyeongnam National University of Science and Technology, Jinju 52725, Korea
}

\section{ABSTRACT}

This study was conducted to analyze the current state and symbolic meanings of floral emblem designation in local governments of South Korea. The scope of local governments subject to analysis was limited to special city, metropolitan city, province, self-governing province, city, self-governing city, county and district based on their administrative divisions. The floral emblems of local governments and their symbolic meanings as of 2019 were examined. A total of 44 plant species were designated as floral emblems. Many plant species with high designation frequency were included in Rhododendron spp., Rosa spp., Camellia spp., Magnolia spp., and Prunus spp.. Plant species with higher designation frequency tended to have more symbolic meanings. A total of 155 terms were used for the symbolic meanings assigned to all the designated floral emblems. The major symbolic meanings were relevant to material affluence or economic growth, community spirit, and personality generally required from local residents. Most of the plant species linked to the top 10 most frequently assigned terms in symbolic meaning were those of the top 10 most frequently designated floral emblems. In the case of floral emblems with high designation frequency, it was shown that they were linked with various symbolic meanings in order to grant identity with regional distinctiveness and differentiation, regardless of the symbolic meaning that the designated flowers generally have. However, the floral emblems with low designation frequency seem to have relatively strong physical or emotional relevance with local governments and thus are expected to have high utilization in regional branding and tourism marketing.

Keywords: city flower, floral trees, local government, national flower, regional identity

\section{Introduction}

Gardens, which have long provided the beauty of nature and recreation for people, have now become major tourist sites. For example, the Suncheonman National Garden opened in 2014 after hosting the Suncheon Bay Garden EXPO 2013. The total number of visitors in 2017 was $6,117,633$ persons $(6,098,849$ natives and 18,784 foreigners), which is remarkably higher than historic sites such as Naganeupseong Walled Town, Songgwangsa Temple, and Seonamsa Temple in Suncheon (Kim and Huh, 2019; Korean Statistical Information Service. 2019). Wyse Jackson and Sutherland (2000) reported that the number of visitors in public gardens worldwide was 150 million as of 2000, and Benfield (2013) estimated that the number of visitors who visited private gardens through open houses operated by institutes such as the Garden Conservancy (USA) and National Gardens Scheme (UK and Australia) and the number of visitors in gardens run by nonprofit organizations like the National Trust (UK) add up to approx-

This work was supported by Gyeongnam National University of Science and Technology Grant in 2018-2019.

Received: January 19, 2020, Revised: January 29, 2020, Accepted: February 6, 2020

First author: Inhea Kim, inhyekim@hanmail.net, (1) https://orcid.org/0000-0001-9915-1623

*Corresponding author: Kyoung-Ok Choi, choiok@gntech.ac.kr, (i) https://orcid.org/0000-0001-7990-7606 
imately 300 million. As such, many people are enjoying garden tourism both locally and globally, but there is insufficient research on garden tourism and its importance is not receiving attention even in tourism, which is the world's biggest industry (Benfield, 2013; Connell, 2004).

Flowers, a major component of gardens, is a symbol of beauty that is constantly been perceived as an aesthetic object throughout the history of humanity, and it is one of the motifs most frequently used in formative arts (Chang, 2016). For a long time, flowers have been a symbol or an icon that expresses various emotions and represents physical and psychological state. Thus, flower designers sublimate or commercialize the beauty and symbolism of flowers into art, provide education to the community, present floral designs in flower shows, weddings and funerals, and use them in horticultural therapy (Chu et al., 2018). In the South Korean design industry, to not only inherit and develop traditional culture but also lead to development of the tourism industry by promoting the originality and excellence of South Korean culture, there has been constant research on design development using traditional floral patterns such as plum blossom, peony, and lotus flower (Chang, 2016; Hong and Kim, 2011; Jung, 2004; Kim, 2009; Song, 2010). Moreover, many countries in the world are designating floral emblems representing the national or regional characteristics and sentiments, which contribute to regional branding and commercialization of tourism resources. The traditional wreath called 'Lei' in Hawaii, a world-renowned tourist site, has a regional meaning depending on the type of flower, and it is known to be a case that has performed an effective role in the tourism industry by applying the culture of presenting the lei to visitors (Bird et al., 1987; Criley, 1992). In South Africa, bird of paradise flower is used to represent the identity of a new nation that integrated multiple ethnicities, and it is perceived as the best icon of tourism advertising for South Africa (Benfield, 2013).

Through a case study on fashionable culture, Goss (1993) suggested that the prominent leisure activities in the modern lifestyle are visiting malls or going shopping, whereas the prominent symbol of attachment is flowers. Ziegler (2007) studied the traces of the changing tastes and traditions in the floricultural culture and industry in the sociological view, discussed the role of flowers in terms of joy, social status and creation of the sense of belonging, and led to the conclusion that one of the sources of various horticultural activities widely in fashion today is creation of identity. In sum, designating a flower as a national or regional symbol can help its members perceive the important symbolic meaning, give them an identity, and cultivate community spirit. Furthermore, floral emblem designation may bring economic benefits to the national and regional community as an element of garden tourism or design. Therefore, this study is conducted to expand the scope of floral emblem designation and use that can increase regional competitiveness by analyzing the current state of floral emblem designation in South Korean local governments and their symbolic meaning, and to provide basic data to come up with designs that reflect regional identity and commercialize the emblems for tourism.

\section{Research Methods}

\section{Research perspectives}

Flowers have a symbolic meaning formed in history and culture along with the external beauty that physically shows, and are used in many countries as a national or regional symbol. Floral emblem designation and symbolic meaning can be understood through the rose of Sharon (Hibiscus syriacus L.), which is the flower that symbolizes South Korea. A national symbol is described as an official symbol represented by images, texts or figures that are distinctive, differentiated and relatable to promote the consistent national identity to the global society while also having citizens perceive a unified national identity and cultivate community spirit. The South Korean national symbols include Taegeukgi (national flag), Aegukga (national anthem), rose of Sharon (national flower), great seal (seal of state), national emblem, etc. The rose of Sharon, which has been loved in South Korean gardens for a long time, indicates an "eternal blossom that never fades". Due to its meaning and emotional communion, it is designated as the national flower that symbolizes the toughness of our people that have overcome foreign invasion due to its geographical position 
of a peninsula (Ministry of the Interior and Safety, 2019). South Africa designated king protea (Protea cynaroides) as the national flower in 1976, but later, the bird of paradise flower (Strelitzia reginae) was used to newly depict the nature of the nation that integrated multiple ethnicities, and it is now perceived as an icon that appears more frequently in the tourism advertisements in South Africa (Benfield, 2013). Based on the context of these cases, research on floral emblem designation and analysis of symbolic meaning is premised on the two following perspectives. First, like the case of the rose of Sharon, the species that has formed the perception of beauty connected to history and culture and that has formed intimate sentiment among flowers that have inhabited the region for a long time as native species is first designated as the floral emblem, after which it is given the symbolic meaning. Second, like the case of the bird of paradise flower, the symbolic meaning that coincides with the goal, identity and community spirit pursued by the local government is first selected, after which the suitable plant species is chosen.

From the first perspective, we investigated the frequency of plants designated as floral emblems by the local governments, assessed the preference tendency and rarity, and analyzed the symbolic meaning given in the process of floral emblem designation for plant species with high designation frequency. From the second perspective, we investigated the frequency of the symbolic meaning given to the floral emblem, assessed the preference tendency and specificity of the symbol, and analyzed the plant species assigned to the symbolic meaning with high frequency. Through the results of analysis in these two aspects, we comprehensively assessed floral emblem designation and symbolic meaning.

\section{Survey and analysis}

South Korean local governments are divided largely into 17 provincial-level administrative divisions such as Seoul special city, Sejong special self-governing city, eight provinces (Gangwon-do, Gyeonggi-do, Chungcheongbuk-do, Chungcheongnam-do, Gyeongsangbuk-do, Gyeongsangnam-do, Jeollabuk-do, Jeollanam-do), six metropolitan cities (Incheon, Daejeon, Daegu, Ulsan, Gwangju, Busan), and Jeju special self-governing province. More specifically, they are sub- divided into municipal-level administrative divisions such as cities (si), counties (gun), and districts (gu). Most provinces, cities, counties and districts have designated symbolic flowers. Thus, the subjects of this study was limited to the provincial-level administrative divisions and the municipal-level administrative divisions, and the current state of floral emblem designation in total 245 local governments was investigated. Data was collected from November 20, 2018 to June 8, 2019 through the website of each local government and phone interviews with the relevant staff. Frequency analysis was conducted on the collected data with focus on whether a floral emblem is designated in each administrative division, the designated plant species, and its symbolic meaning. Even though the floral emblem is designated in the provincial-level administrative division, a separate floral emblem is designated again in the municipal-level administrative division. Thus, the floral emblem designation frequency was calculated independently in all local governments regardless of the hierarchy of the administrative division. To discuss from the first research perspective, we analyzed the types of plants designated as the floral emblems as well as the frequency, after which we analyzed the symbolic meanings given to the top 10 plant species in designation frequency. To discuss from the second research perspective, we drew terms used as the symbolic meanings of the floral emblems and conducted a frequency analysis, after which we analyzed the plant species assigned to the top 10 terms, and comprehensively discussed the results in the two aspects.

\section{Results and Discussion}

The total floral emblem designation rate of the 245 local governments examined in this study was $97.6 \%$, indicating that most local governments were designating their own floral emblems in general (Table 1). However, the floral emblem designation rate of Daejeon metropolitan city was 33.3\%, which was remarkably lower than other local governments. The floral emblem designation rates of Incheon metropolitan city, Busan metropolitan city, and Gyeonggi-do province were $90.9 \%, 94.1 \%$ and $93.8 \%$ respectively, showing that some local governments in the administrative divisions did 
Table 1. Floral emblem designation in the local governments of South Korea

\begin{tabular}{|c|c|c|c|c|c|}
\hline $\begin{array}{l}\text { Provincial-level } \\
\text { administrative } \\
\text { divisions }\end{array}$ & $\begin{array}{l}\text { Municipal-level } \\
\text { administrative } \\
\text { divisions }\end{array}$ & $\begin{array}{l}\text { Number of } \\
\text { administrative } \\
\text { divisions }\end{array}$ & $\begin{array}{c}\text { Floral emblem } \\
\text { designation } \\
\text { frequency }\end{array}$ & $\begin{array}{c}\text { Floral emblem } \\
\text { designation rate } \\
(\%)\end{array}$ & $\begin{array}{l}\text { Number of plant } \\
\text { species designated } \\
\text { as floral emblem }\end{array}$ \\
\hline Seoul special city & 25 districts & 26 & 26 & 100.0 & 12 \\
\hline Incheon metropolitan city & $\begin{array}{l}8 \text { districts } \\
2 \text { counties }\end{array}$ & 11 & 10 & 90.9 & 6 \\
\hline Daejeon metropolitan city & 5 districts & 6 & 2 & 33.3 & 2 \\
\hline Gwangju metropolitan city & 5 districts & 6 & 6 & 100.0 & 3 \\
\hline Daegu metropolitan city & $\begin{array}{l}7 \text { districts } \\
1 \text { county }\end{array}$ & 9 & 9 & 100.0 & 7 \\
\hline Ulsan metropolitan city & $\begin{array}{l}4 \text { districts } \\
1 \text { county }\end{array}$ & 6 & 6 & 100.0 & 5 \\
\hline Busan metropolitan city & $\begin{array}{l}15 \text { districts } \\
1 \text { county }\end{array}$ & 17 & 16 & 94.1 & 10 \\
\hline Sejong special self-governing city & - & 1 & 1 & 100.0 & 1 \\
\hline Gyeonggi-do province & $\begin{array}{c}28 \text { cities } \\
3 \text { counties }\end{array}$ & 32 & 30 & 93.8 & 12 \\
\hline Gangwon-do province & $\begin{array}{c}7 \text { cities } \\
11 \text { counties }\end{array}$ & 19 & 19 & 100.0 & 11 \\
\hline Chungcheongbuk-do province & $\begin{array}{c}3 \text { cities } \\
8 \text { counties }\end{array}$ & 12 & 13 & 108.3 & 8 \\
\hline Chungcheongnam-do province & $\begin{array}{c}8 \text { cities } \\
7 \text { counties }\end{array}$ & 16 & 16 & 100.0 & 8 \\
\hline Jeollabuk-do province & $\begin{array}{c}6 \text { cities } \\
8 \text { counties }\end{array}$ & 15 & 15 & 100.0 & 7 \\
\hline Jeollanam-do province & $\begin{array}{c}5 \text { cities } \\
17 \text { counties }\end{array}$ & 23 & 24 & 104.3 & 13 \\
\hline Gyeongsangbuk-do province & $\begin{array}{c}10 \text { cities } \\
13 \text { counties }\end{array}$ & 24 & 24 & 100.0 & 13 \\
\hline Gyeongsangnam-do province & $\begin{array}{c}8 \text { cities } \\
10 \text { counties }\end{array}$ & 19 & 19 & 100.0 & 13 \\
\hline Jeju special self-governing province & 2 cities & 3 & 3 & 100.0 & 3 \\
\hline Total & & 245 & 239 & 97.6 & \\
\hline
\end{tabular}

${ }^{\mathrm{z}}$ Provincial-level administrative divisions are subdivided into municipal-level administrative divisions including cities (si), counties (gun), and districts (gu).

${ }^{\mathrm{y}}$ Number of administrative divisions is calculated as the total sum of the number of provincial-level and municipal-level administrative divisions. ${ }^{\mathrm{x}}$ Floral emblem designation rate is calculated by (floral emblem designation frequency/number of administrative divisions) $\times 100$.

not designate floral emblems. The floral emblem designation rates of Chungcheongbuk-do province and Jeollanam-do province were $108.3 \%$ and $104.3 \%$ respectively, indicating that some local governments in the administrative divisions designated two or more floral emblems.

Seoul special city, the capital of South Korea, was designating floral emblems, and 25 districts in the administrative division were independently designating floral emblems.
However, 12 types of flowers were designated as floral emblems, indicating that local governments were designating the same plant species as the floral emblems. Incheon metropolitan city and Gyeonggi-do province in the capital area also showed a similar tendency. Jeju special self-governing province showed a $100 \%$ floral emblem designation rate, and in total three local governments (Jeju special self-governing province in the provincial-level and two cit- 
ies in the municipal-level administrative division), there were three types of flowers designated as the floral emblems, thereby not overlapped. Sejong special self-governing city was designating one floral emblem. Among the metropolitan cities, Busan metropolitan city had 10 types of plants designated as the floral emblems, showing the greatest variety, and among the provinces, Jeollanam-do province, Gyeongsangbuk-do province, and Gyeongsangnam-do province had 13 types, showing the greatest variety of plants. The types of plants designated as floral emblems were more diverse in the southern region than the central region, due to the climatic features and extensive flora of the southern region where not only temperate plants but also warm temperate plants can grow.

Total 44 plant species were designated by the local governments as their floral emblems as shown in Table 2, which did not include rose of Sharon, the national flower of South Korea. The plant species of floral emblems with high designation frequency were mostly flowering trees or shrubs, including Rhododendron spp., Rosa spp., Camellia spp., Magnolia spp., Prunus spp., etc. They were plant species popular worldwide and thus easily found in botanical gardens or parks in not only South Korea but also overseas. Among them, Rhododendron spp., Camellia spp., Magnolia spp., and Prunus spp. are known as plant species that are produced extensively as landscaping materials in South Korea (Korea Forest Service, 2007). On the other hand, plant species of floral emblems with low designation frequency included relatively more perennial herbaceous flowering plants. The designation rates of the top 5 species such as Rhododendron schlippenbachii Max. (17.4\%), Forsythia koreana Nakai. (13.0\%), Camellia japonica L. (8.9\%), Rhododendron mucronulatum Turcz. (7.7\%), and Rosa hybrida Hort. (7.3\%) added up to $54.3 \%$, indicating that floral emblem designation was concentrated in certain plant species. This result is similar to that of previous research in which city flower designation was concentrated in Forsythia koreana Nakai., Rhododendron schlippenbachii Max., Camellia japonica L., and Rosa hybrida Hort. (Rho, 1998).

In particular, Forsythia koreana Nakai. and Rhododendron mucronulatum Turcz., the native habitat of which is South Korea, showed the second-highest and fourth-highest des- ignation frequency respectively. Forsythia koreana Nakai. and Rhododendron mucronulatum Turcz. are flowering shrubs that grow in various places of South Korea in spring, and they are native plants that have provided deep emotional connection throughout the history and culture of South Korea, which is why many local governments have designated them as the floral emblems. Bang and Lee (1995) who analyzed the plantation distribution of landscaping trees in South Korea also proved that Forsythia koreana Nakai. and Rhododendron mucronulatum Turcz. showed extensive plantation distribution. Moreover, Camellia japonica L. is a flowering tree that grow only in the southern region but not in the central region of South Korea, and its flowers create the exotic and unique landscape of the southern region, which is why many local governments in the southern region have designated Camellia japonica L. as the floral emblem to differentiate themselves from the central region.

Floral emblems with low designation frequency tended to show relatively high physical/emotional correlation with local governments. Yeongdeok-gun county, where peach is its regional specialty, designated Prunus persica L. as the floral emblem, whereas Cheongsong-gun county that is a famous apple producer designated Malus pumila Miller as the floral emblem, which shows the correlation between floral emblem designation and regional specialty. Moreover, Abeliophyllum distichum Nakai, which is a world-renowned rare plant that naturally grows only in South Korea, was designated as the floral emblem of Goesan-gun county where the plant was first discovered. Pulsatilla tongkangensis Y.N. Lee \& T.C. Lee (Donggang pasqueflower) was designated as the floral emblem of Jeongseon-gun county, which is its native habitat. Jeongseon-gun county not only designated Pulsatilla tongkangensis Y.N. Lee \& T.C. Lee as its floral emblem but also designated the Donggang Pasqueflower Village, hosting the Jeongseon Donggang Pasqueflower Festival at the end of March every year (Jeongseon, 2019). These cases prove the strong connectivity between floral emblems and regional characteristics.

As a result of analyzing the symbolic meanings given to the plant species with the top 10 highest designation frequency to analyze the symbolic meanings of floral emblems, it was found that 40 symbolic meanings were given 
Table 2. Plant species ranks based on floral emblem designation frequency

\begin{tabular}{|c|c|c|c|c|}
\hline Rank & $\begin{array}{l}\text { Plant species designated } \\
\text { as floral emblem }\end{array}$ & Administrative division & Frequency & $\begin{array}{l}\text { Percentage } \\
\quad(\%)\end{array}$ \\
\hline 1 & $\begin{array}{l}\text { Rhododendron schlippenbachii } \\
\text { Max. }\end{array}$ & $\begin{array}{l}\text { Boseong-gun, Buan-gun, Busan Buk-gu, Busanjin-gu, } \\
\text { Cheolwon-gun, Cheongdo-gun, Cheongyang-gun, Daegu Dong-gu, } \\
\text { Danyang-gun, Gangwon-do, Gokseong-gun, Goryeong-gun, } \\
\text { Guri-si, Gurye-gun, Gwacheon-si, Gwanak-gu, Gwangju Dong-gu, } \\
\text { Gwangju metropolitan city, Gwangju Nam-gu, Gyeryong-si, } \\
\text { Hadong-gun, Hamyang-gun, Incheon Dong-gu, Incheon Jung-gu, } \\
\text { Inje-gun, Jinan-gun, Jongro-gu, Milyang-si, Muju-gun, } \\
\text { Mungyeong-si, Namwon-si, Pyeongchang-gun, Sacheon-si, } \\
\text { Samcheok-si, Sasang-gu, Seongnam-si, Suncheon-si, Uijeongbu-si, } \\
\text { Uiwang-si, Wanju-gun, Yeongju-si, Yeongwol-gun, Youngin-si }\end{array}$ & 43 & 17.4 \\
\hline 2 & Forsythia koreana Nakai. & $\begin{array}{l}\text { Anseong-si, Anyang-si, Boeun-gun, Cheonan-si, Chuncheon-si, } \\
\text { Daegu Nam-gu, Eumseong-gun, Gapyeong-gun, Geochang-gun, } \\
\text { Gongju-si, Gumi-si, Gunwi-gun, Gwangju-si, Gyeonggi-do, } \\
\text { Gyeongju-si, Heungseong-gun, Imsil-gun, Incheon Namdong-gu, } \\
\text { Jecheon-si, Jeonju-si, Kimpo-si, Namyangju-si, Nonsan-si, } \\
\text { Okcheon-gun, Seongdong-gu, Seongju-gun, Seoul special city, } \\
\text { Songpa-gu, Yangju-si, Yeoju-si, Yeoncheon-gun, Yesan-gun }\end{array}$ & 32 & 13.0 \\
\hline 3 & Camellia japonica L. & $\begin{array}{l}\text { Boryeong-si, Busan Jung-gu, Busan metropolitan city, Gangjin-gun, } \\
\text { Geoje-si, Gochang-gun, Goheung-gun, Gunsan-si, Gwangyang-si, } \\
\text { Haenam-gun, Haeundae-gu, Jeollanam-do, Jindo-gun, } \\
\text { Seocheon-gun, Taean-gun, Tongyeong-si, Ulleung-gun, Ulsan } \\
\text { Dong-gu, Ulsan Jung-gu, Wando-gun, Yeongdo-gu, Yeosu-si }\end{array}$ & 22 & 8.9 \\
\hline 4 & $\begin{array}{l}\text { Rhododendron mucronulatum } \\
\text { Turcz. }\end{array}$ & $\begin{array}{l}\text { Daegu Seo-gu, Dangjin-si, Gangbuk-gu, Ganghwa-gun, } \\
\text { Geumcheon-gu, Geumjeong-gu, Gijang-gun, Gwangjin-gu, } \\
\text { Gyeyang-gu, Hongcheon-gun, Hwacheon-gun, Icheon-si, } \\
\text { Jangheung-gun, Seongbuk-gu, Suwon-si, Uiryeong-gun, } \\
\text { Yangpyeong-gun, Yeongdong-gun, Yeonsu-gu }\end{array}$ & 19 & 7.7 \\
\hline 5 & Rosa hybrida Hort. & $\begin{array}{l}\text { Ansan-si, Dalseo-gu, Goyang-si, Gwangmyeong-si, } \\
\text { Gyeongsangnam-do, Incheon metropolitan city, Jincheon-gun, } \\
\text { Pohang-si, Saha-gu, Sangju-si, Seocho-gu, Seodaemun-gu, Seoul } \\
\text { Jung-gu, Suseong-gu, Ulsan metropolitan city, Wonju-si, } \\
\text { Yeongcheon-si, Yongsan-gu }\end{array}$ & 18 & 7.3 \\
\hline 6 & $\begin{array}{l}\text { Chrysanthemum morifolium } \\
\text { Ram. }\end{array}$ & $\begin{array}{l}\text { Bupyeong-gu, Changyeong-gun, Cheongcheongnam-do, } \\
\text { Chungju-si, Dongjak-gu, Gangseo-gu, Goseong-gun, Gujung-gu, } \\
\text { Iksan-si, Incheon Seo-gu, Muan-gun, Seosan-si, Sokcho-si }\end{array}$ & 13 & 5.3 \\
\hline 7 & Magnolia kobus DC. & $\begin{array}{l}\text { Asan-si, Busan Nam-gu, Busan Seo-gu, Daegu metropolitan city, } \\
\text { Dongdaemun-gu, Gangnam-gu, Guro-gu, Gwangju Seo-gu, } \\
\text { Gwangsan-gu, Gyeongsan-si, Mapo-gu, Yangsan-si, Yecheon-gun, } \\
\text { Yeongdeungpo-gu }\end{array}$ & 14 & 5.7 \\
\hline 8 & Prunus mume Sieb. et Zucc. & $\begin{array}{l}\text { Andong-si, Chilgok-gun, Damyang-gun, Donghae-si, Dongrae-gu, } \\
\text { Gangdong-gu, Hapcheon-gun, Kimhae-si, Osan-si, Uljin-gun, } \\
\text { Yeongam-gun }\end{array}$ & 11 & 4.5 \\
\hline 9 & Zininia elegans Jacq. & $\begin{array}{l}\text { Daegu Jung-gu, Gwangju Buk-gu, Gyeongsangbuk-do, Haman-gun, } \\
\text { Jeollabuk-do, Kimje-si }\end{array}$ & 6 & 2.4 \\
\hline \multirow[t]{2}{*}{10} & Magnolia denudata Desroux. & $\begin{array}{l}\text { Cheongju-si, Chungcheongbuk-do, Daejeon metropolitan city, } \\
\text { Jeungpyeong-gun, Mokpo-si }\end{array}$ & 5 & 2.0 \\
\hline & Pyrus serotina Rehder & Busan Gangseo-gu, Jungrang-gu, Naju-si, Pyeongtaek-si, Ulju-gun & 5 & 2.0 \\
\hline \multirow{2}{*}{11} & Rosa rugosa Thunb. & Goseong-gun, Ongjin-gun, Sinan-gun, Yangyang-gun & 4 & 1.6 \\
\hline & Lagerstroemia indica L. & Daegu Buk-gu, Gangneung-si, Hwaseong-si, Sunchang-gun & 4 & 1.6 \\
\hline
\end{tabular}


Table 2. (continued)

\begin{tabular}{|c|c|c|c|c|}
\hline Rank & $\begin{array}{l}\text { Plant species designated } \\
\text { as floral emblem }\end{array}$ & Administrative division & Frequency & $\begin{array}{c}\text { Percentage } \\
(\%)\end{array}$ \\
\hline \multirow{4}{*}{12} & Cosmos bipinnatus Cav. & Eunpyeong-gu, Gokseong-gun, Paju-si & 3 & 1.2 \\
\hline & Magnolia parviflora Sieb. et Zucc. & Hoengseong-gun, Suyeong-gu, Taebaek-si & 3 & 1.2 \\
\hline & Nelumbo nucifera Gaertn. & Buyeo-gun, Yeongyang-gun, Yeonje-gu & 3 & 1.2 \\
\hline & Prunus persica $\mathrm{L}$. & $\begin{array}{l}\text { Bucheon-si, Sejong special self-governing city, } \\
\text { Yeongdeok-gun }\end{array}$ & 3 & 1.2 \\
\hline \multirow{4}{*}{13} & $\begin{array}{l}\text { Chrysanthemum zawadskii Herbich var. latilobum } \\
\text { (Maxim.) Kitamura }\end{array}$ & Jeongeup-si, Pochen-si & 2 & 0.8 \\
\hline & Prunus serrulata Thunb. var. spontanea Max. Wils. & Changwon-si, Ulsan Nam-gu & 2 & 0.8 \\
\hline & Rhododendron weyrichii Maxim. & Dalseong-gun, Jeju special self-governing province & 2 & 0.8 \\
\hline & $\begin{array}{l}\text { Rhododendron yedoense Maxim. ex Regel var. } \\
\text { poukhanense }\end{array}$ & Jangsu-gun, Nowon-gu & 2 & 0.8 \\
\hline \multirow{25}{*}{14} & Abeliophyllum distichum Nakai & Goesan-gun & 1 & 0.4 \\
\hline & Chrysanthemum indicum $\mathrm{L}$. & Hwasun-gun & 1 & 0.4 \\
\hline & Convallaria keiskei (Miq.) Miquel & Hanam-si & 1 & 0.4 \\
\hline & Cornus officinalis Sieb. et Zucc. & Uiseong-gun & 1 & 0.4 \\
\hline & Cymbidium goeringii (Reichb. fil.) Reichb. fil. & Hampyeong-gun & 1 & 0.4 \\
\hline & Cymbidium kanran Makino & Seogwipo-si & 1 & 0.4 \\
\hline & Gardenia jasminoides Ellis var. grandiflora Nakai & Namhae-gun & 1 & 0.4 \\
\hline & Gossypium indicum Lam. & Sancheong-gun & 1 & 0.4 \\
\hline & Helianthus annuus L. & Yangcheon-gu & 1 & 0.4 \\
\hline & Impatiens balsamina $\mathrm{L}$. & Goesan-gun & 1 & 0.4 \\
\hline & Iris ensata var. spontanea (Makino) Nakai & Dobong-gu & 1 & 0.4 \\
\hline & Kerria japonica (L.) DC. & Dongducheon-si & 1 & 0.4 \\
\hline & Lilium tigrinum Ker-Gawl. & Ulsan Buk-gu & 1 & 0.4 \\
\hline & Lycoris sanguinea var. koreana (Nakai) T. Koyama & Jangseong-gun & 1 & 0.4 \\
\hline & Lycoris squamigera Maxim. & Yeonggwang-gun & 1 & 0.4 \\
\hline & Magnolia sieboldii K. Koch & Bonghwa-gun & 1 & 0.4 \\
\hline & Malus pumila Miller & Cheongsong-gun & 1 & 0.4 \\
\hline & Narcissus spp. & Jeju-si & 1 & 0.4 \\
\hline & Paeonia suffruticosa Andr. & Geumsan-gun & 1 & 0.4 \\
\hline & Prunus armeniaca L. var. ansu Max. & Yanggu-gun & 1 & 0.4 \\
\hline & Prunus salicina Lindl. & Gimcheon-si & 1 & 0.4 \\
\hline & Pulsatilla tongkangensis Y. N. Lee \& T. C. Lee & Jeongseon-gun & 1 & 0.4 \\
\hline & Punica granafum L. & Jinju-si & 1 & 0.4 \\
\hline & No floral emblem & $\begin{array}{l}\text { Busan Dong-gu, Daejeon Daedeok-gu, Daejeon } \\
\text { Dong-gu, Daejeon Seo-gu, Gunpo-si, Michuhol-gu, } \\
\text { Siheung-si, Yuseong-gu }\end{array}$ & 8 & 3.2 \\
\hline & Total & & 247 & 100.0 \\
\hline
\end{tabular}

to Rhododendron schlippenbachii Max., at least 20 meanings to Forsythia koreana Nakai., Camellia japonica L., Rhododendron mucronulatum Turcz., Rosa hybrida Hort., and Magnolia kobus DC., and at least 10 to other plant species. Plant species with higher designation frequency tended to have more symbolic meanings (Table 3). Compared 
Table 3. Symbolic meanings of the top ten designation ranking floral emblems

\begin{tabular}{|c|c|c|c|}
\hline $\begin{array}{l}\text { Designation } \\
\text { rank }\end{array}$ & $\begin{array}{c}\text { Floral } \\
\text { emblem }\end{array}$ & $\begin{array}{l}\text { Number of } \\
\text { meanings }\end{array}$ & $\begin{array}{l}\text { Symbolic meanings } \\
\text { (Assignment frequency) }\end{array}$ \\
\hline 1 & $\begin{array}{l}\text { Rhododendron } \\
\text { schlippenbachii Max. }\end{array}$ & 40 & $\begin{array}{l}\text { Prosperity(7), Mental(5), Vigor(5), Combination(4), Love(4), Patience(4), Richness(4), } \\
\text { Fidelity(3), Amiable(2), Beauty(2), Development(2), Endurance(2), Joy(2), Local } \\
\text { patriotism(2), Passion(2), Politeness(2), Pure(2), Strength(2), Wise(2), Citizenship(1), } \\
\text { Clarity(1), Cohesion(1), Concession(1), Democratization(1), Exuberancy(1), Faith(1), } \\
\text { Familiarity(1), Foreknowledge(1), Growth(1), Honor(1), Hospitality(1), Industry(1), } \\
\text { Loyalty(1), Moderation(1), Pioneer(1), Potential(1), Recognition(1), Rusticity(1), } \\
\text { Tender(1), Youth(1) }\end{array}$ \\
\hline 2 & $\begin{array}{l}\text { Forsythia koreana } \\
\qquad \text { Nakai }\end{array}$ & 25 & $\begin{array}{l}\text { Hope(10), Prosperity(7), Cohesion(4), Development(3), Fighting spirit(3), Vigor(3), } \\
\text { Cooperation(2), Friendly(2), Patience(2), Peace(2), Unsophisticated(2), Assiduity(1), } \\
\text { Combination(1), Desire(1), Future(1), Hospitality(1), Light(1), Liveliness(1), Love(1), } \\
\text { Pioneer(1), Positive thinking(1), Power(1), Stability(1), Tidings of spring (1), Virtue(1) }\end{array}$ \\
\hline 3 & Camellia japonica L. & 27 & $\begin{array}{l}\text { Love(3), Prosperity(3), Vigor(3), Passion(2), Sea(2), Will(2), Backbone(1), Beach(1), } \\
\text { Beauty(1), Chastity(1), Cohesion(1), Combination(1), Courage(1), Endurance(1), } \\
\text { Exuberancy(1), Friends of Korean traditional scholar(1), Fruition(1), Hope(1), } \\
\text { Industry(1), Local patriotism(1), Luck(1), Patience(1), Potential(1), Rusticity(1), } \\
\text { Strength(1), Talent producing(1), Victory(1) }\end{array}$ \\
\hline 4 & $\begin{array}{l}\text { Rhododendron } \\
\text { mucronulatum Turcz. }\end{array}$ & 20 & $\begin{array}{l}\text { Cohesion(5), Combination(5), Rusticity(4), Industry(3), Love(3), Familiarity(1), Filial } \\
\text { duty(1), Hope(1), Independence(1), Iron hand in a velvet glove (1), Life force(1), Local } \\
\text { patriotism(1), Mind(1), Moderation(1), Mountain filled with azalea(1), Personality(1), } \\
\text { Prosperity(1), Strength(1), Tender(1), Warmth(1) }\end{array}$ \\
\hline 5 & Rosa hybrida Hort. & 21 & $\begin{array}{l}\text { Passion(5), Beauty(4), Courage(3), Love(3), Prosperity(3), Wisdom(3), } \\
\text { Development(2), Local patriotism(2), Active(1), Chastity(1), Combination(1), } \\
\text { Concord(1), Dignity(1), Elegance(1), Enthusiasm(1), Fragrant(1), Future(1), Grace(1), } \\
\text { Longing(1), Possibility(1), Proud(1) }\end{array}$ \\
\hline 6 & $\begin{array}{l}\text { Chrysanthemum } \\
\text { morifolium Ram. }\end{array}$ & 17 & $\begin{array}{l}\text { Richness(4), Patience(2), Prosperity(2), Stability(2), Vigor(2), Constancy(1), } \\
\text { Development(1), Fidelity(1), Friendly(1), Loyalty(1), Mild(1), Personality(1), } \\
\text { Politeness(1), Pure(1), Sophisticated(1), Tender(1) }\end{array}$ \\
\hline 7 & Magnolia kobus DC. & 23 & $\begin{array}{l}\text { Chastity(2), Cohesion(2), Grace(2), Heart(2), Mental(2), Neat(2), Capacity(1), } \\
\text { Combination(1), Decent(1), Elegance(1), Favor(1), Love(1), Peace(1), Pure(1), } \\
\text { Recognition(1), Richness(1), Sacrifice(1), Smooth(1), Stability(1), Thermal water(1), } \\
\text { Unsophisticated(1), Verdure(1), Will(1) }\end{array}$ \\
\hline 8 & $\begin{array}{l}\text { Prunus mume Sieb. et } \\
\text { Zucc. }\end{array}$ & 17 & $\begin{array}{l}\text { Noble(4), Fidelity(2), Strength(2), Backbone(1), Creation(1), Development(1), } \\
\text { Endurance(1), Grace(1), Heart(1), Humility(1), Moral character(1), Morality(1), } \\
\text { Personality(1), Prosperity(1), Seonbijeongsin (Korean traditional scholar spirit)(1), } \\
\text { Self-help(1), Virtue(1) }\end{array}$ \\
\hline 9 & Zininia elegans Jacq. & 11 & $\begin{array}{l}\text { Patience(4), Endurance(2), 100-day blooming(1), Development(1), Familiarity(1), } \\
\text { Force(1), Grace(1), Mental(1), Potential(1), Vigor(1), Will(1) }\end{array}$ \\
\hline \multirow[b]{2}{*}{10} & $\begin{array}{l}\text { Magnolia denudata } \\
\text { Desroux. }\end{array}$ & 11 & $\begin{array}{l}\text { Chastity(2), Art(1), Combination(1), Cooperation(1), Decent(1), Elegance(1), } \\
\text { Mental(1), Mild(1), Naive(1), Self-help(1), Self-reliance(1) }\end{array}$ \\
\hline & Pyrus serotina Rehder & 11 & $\begin{array}{l}\text { Unsophisticated(2), Assiduity(1), Backbone(1), Beauty(1), Cohesion(1), Creation(1), } \\
\text { Ethnicity(1), Hope(1), Recognition(1), Seonbijeongsin(Korean traditional scholar } \\
\text { spirit)(1), Will(1) }\end{array}$ \\
\hline
\end{tabular}

to the fact that each flower generally has 2-5 commonly used languages of flowers, there were extremely diverse symbolic meanings given to the plant species with the top 10 highest floral emblem designation frequency. Most flo- ral emblems were given at least 2 symbolic meanings, and a comparison of symbolic meanings given by multiple local governments to the same floral emblem shows that not only the same or similar terms but also special and distinctive 
terms were used. This result can be analyzed in line with the argument by Ziegler (2007) that "creation of identity is an activity fundamental to several of today's widespread flower practices". In terms of establishing an identity distinguished from other regions and giving the sense of belonging or homogeneity, even if the members of the community agreed to designate the universally preferred plants such as Rhododendron schlippenbachii Max., Forsythia koreana Nakai., Rosa hybrida Hort., and Camellia japonica L. as the floral emblems, it seems that differentiated identity is attempted to be given to those plants in terms of symbolic meaning. This indicates that, since the same plant species can be perceived with different emotions, symbolic meanings of floral emblems are used to establish an identity among regions with diverse historical and cultural backgrounds.

Regarding the symbolic meanings of floral emblems, some local governments have given symbolic meanings with commonly used floriography to the flowers designated as the floral emblems, while others have given symbolic meanings with special historical/cultural sentiments or pursued values regardless of floriography. Rhododendron schlippenbachii Max. was given 40 symbolic meanings, which was the biggest number, and 'joy' and 'passion' were equivalent or similar to the widely used floriography, whereas 'prosperity', 'richness', 'local patriotism', and 'citizenship' were values pursued by the local governments. Other emotional meanings seemed to have reflected the special historical/cultural sentiments of the region. Rosa hybrida Hort. was given 25 symbolic meanings, which was approximately $50 \%$ lower than Rhododendron schlippenbachii Max., and the meanings were mostly equivalent or similar to the widely used floriography, while some local governments gave symbolic meanings of 'prosperity', 'local patriotism', 'development', and 'concord'. Among the symbolic meanings of Camellia japonica L., 'sea' seems to be correlated with the distinctive marine landscape of the southern Korean region, which is the habitat of Camellia japonica L. Symbolic meanings such as 'iron hand in a velvet glove' given to Rhododendron mucronulatum Turcz., 'fidelity' and 'Seonbijeongsin (Korean traditional scholar spirit)' given to Prunus mume Sieb. et Zucc., and 'ethnicity' given to Pyrus serotina Rehder showed correla- tion to historical/cultural sentiments.

Total 155 terms were used as symbolic meanings of floral emblems, with 'prosperity' showing the highest frequency (23 times), followed by 'cohesion' and 'combination' (Table 4). Major terms used as symbolic meanings were related to material affluence and economic aspects such as 'prosperity', 'development', 'exuberancy', 'richness', and 'growth'; local community spirit such as 'cohesion', 'combination', 'cooperation', 'citizenship', and 'local patriotism'; and personality generally required from local residents such as 'love', 'patience', 'hope', and 'peace'. Terms such as 'Bulgap mountain', 'herbal town', 'first discovered', 'the first plantation', 'beach', 'thermal water', and 'mountain filled with azalea' represented symbolic meanings that are strongly connected to the regional landscapes or features. Moreover, terms that represented the traditional value system or Confucian ideas of Korea such as 'Seonbijeongsin (Korean traditional scholar spirit)' or 'friends of Korean traditional scholar' were also used as symbolic meanings.

Table 5 shows the plant species associated with the terms with the top 10 frequency in symbolic meanings, most of which were plant species included in the top 10 floral emblem designation frequency (Tables 2 and 3). 'Prosperity' was associated with 9 plant species, and seven of them excluding Kerria japonica (L.) DC. and Lilium tigrinum Ker-Gawl. were included in the ones with the top 10 floral emblem designation frequency. 'Cohesion' was associated with 10 plant species, among which Forsythia koreana Nakai., Magnolia kobus DC., Pyrus serotina Rehder, and Rhododendron schlippenbachii Max. were the plant species included among the top 10 designation frequency. 'fidelity', 'noble', and 'peace' that were among the top 10 frequency in symbolic meanings also showed a similar tendency. This result is consistent with the results that local governments designated plant species with high historical/cultural intimacy as the floral emblems (Table 2), that the symbolic meanings given to the floral emblems were more associated with various terms that reveal the differentiated identity of the local governments than the common floriography (Table 3), and that the symbolic meanings related to regional economy, local community spirit, and personality required from local residents were highly perceived (Table 4). 
Table 4. Symbolic meaning of floral emblem ranked by the semantic assignment frequency of administrative divisions

\begin{tabular}{|c|c|c|c|c|c|c|}
\hline \multirow{2}{*}{$\begin{array}{c}\text { Rank } \\
1\end{array}$} & \multirow[t]{2}{*}{ Symbolic meaning of floral emblem } & \multirow{2}{*}{$\begin{array}{c}\text { Number of } \\
\text { meanings } \\
\text { within the } \\
\text { same rank } \\
1\end{array}$} & \multicolumn{2}{|c|}{$\begin{array}{l}\text { Assignment } \\
\text { frequency of } \\
\text { each meaning } \\
\text { (Percent) }\end{array}$} & \multicolumn{2}{|c|}{$\begin{array}{l}\text { Subtotal } \\
\text { assignment } \\
\text { frequency }^{\mathrm{z}} \\
\text { (Percent) }\end{array}$} \\
\hline & & & 23 & $(5.3 \%)$ & 23 & $(5.3 \%)$ \\
\hline 2 & Cohesion & 1 & 19 & $(4.3 \%)$ & 19 & $(4.3 \%)$ \\
\hline 3 & Combination & 1 & 18 & $(4.1 \%)$ & 18 & $(4.1 \%)$ \\
\hline 4 & Love & 1 & 17 & $(3.9 \%)$ & 17 & $(3.9 \%)$ \\
\hline 5 & Mental, Patience, Vigor & 3 & 13 & $(3.0 \%)$ & 39 & $(8.9 \%)$ \\
\hline 6 & Hope, Passion, Richness & 3 & 10 & $(2.3 \%)$ & 30 & $(6.9 \%)$ \\
\hline 7 & Development, Personality, Will & 3 & 8 & $(1.8 \%)$ & 24 & $(5.5 \%)$ \\
\hline 8 & Beauty, Chastity, Local patriotism, Strength & 4 & 7 & $(1.6 \%)$ & 28 & $(6.4 \%)$ \\
\hline 9 & Industry, Unsophisticated & 2 & 6 & $(1.4 \%)$ & 12 & $(2.7 \%)$ \\
\hline 10 & Fidelity, Noble, Peace & 3 & 5 & $(1.1 \%)$ & 15 & $(3.4 \%)$ \\
\hline 11 & Backbone, Courage, Endurance, Rusticity, Stability & 5 & 4 & $(0.9 \%)$ & 20 & $(4.6 \%)$ \\
\hline 12 & $\begin{array}{l}\text { Cleanliness, Enthusiasm, Ethos, Fighting spirit, Friendly, Grace, Heart, } \\
\text { Joy, Luck, Mild, Moderation, Politeness, Potential, Pure, Recognition, } \\
\text { Tender, Wise, Youth }\end{array}$ & 18 & 3 & $(0.7 \%)$ & 54 & $(12.4 \%)$ \\
\hline 13 & $\begin{array}{l}\text { Amiable, Aspiration, Autogenous, Citizenship, Desire, Dignity, } \\
\text { Elegance, Emotion, Ethnicity, Exuberancy, Familiarity, Figure, Fruition, } \\
\text { Future, Life force, Neat, Patiently, Pioneer, Seonbijeongsin(Korean } \\
\text { traditional scholar spirit), Sea, Virtue, Warmth }\end{array}$ & 22 & 2 & $(0.5 \%)$ & 44 & $(10.1 \%)$ \\
\hline 14 & $\begin{array}{l}\text { 100-day blooming, Active, Appearance, Art, Aspect, Assiduity, Beach, } \\
\text { Bulgap Mountain, Capacity, Charm, Cheerful, Clarity, Concession, } \\
\text { Concord, Constancy, Cooperation, Creation, Decent, Democratization, } \\
\text { Dream, Energy, Faith, Favor, Filial duty, First discovered, First } \\
\text { plantation, Foreknowledge, Fragrant, Friends of Korean traditional } \\
\text { scholar, Generosity, Growth, Gumption, Harmony, Herbal town, } \\
\text { Holiness, Honor, Hospitality, humility, Independence, Innocence, Iron } \\
\text { hand in a velvet glove, Jump, Knowledge-morals-body, Life, Light, } \\
\text { Liveliness, Living, Longing, Loyalty, Lustiness, Lyrical meaning, Mind, } \\
\text { Moral character, Morality, Mountain filled with azalea, Naive, News, } \\
\text { Noble man, Order, Origin, Patriotic loyalty, Patriotic spirit, Plain, Pledge, } \\
\text { Positive thinking, Possibility, Power, Pride, Proud, Resident character, } \\
\text { Sacrifice, Self-help, Self-reliance, Smooth, Sophisticated, Stamina, } \\
\text { Status, Talent producing, Temperament, Thermal water, Tidings of } \\
\text { spring, Time, Utility, Verdure, Viability, Victory, Vitality, Wisdom }\end{array}$ & 88 & 1 & $(0.2 \%)$ & 88 & $(20.1 \%)$ \\
\hline & No symbolic meaning & - & & - & 6 & $(1.4 \%)$ \\
\hline & Total & 155 & & & 437 & $(100.0 \%)$ \\
\hline
\end{tabular}

${ }^{\mathrm{z}}$ Subtotal assignment frequency is calculated by (number of meanings within the same rank) $\times($ assignment frequency of each meaning).

\section{Conclusion}

As a symbol or an icon designated by the national or regional community, floral emblems can not only provide members with an identity through important symbolic meanings and cultivate community spirit, but also bring economic benefits to the national and local governments as a component of tourism or design. This study was conducted to analyze the current state of floral emblem designation in the South Korean local governments as well as the symbolic meanings as part of research to promote floral emblem designation and utilization. In general, most local 
Table 5. Plant species frequency of the top ten assignment ranking symbolic meanings

\begin{tabular}{|c|c|c|c|}
\hline $\begin{array}{l}\text { Assign } \\
\text {-ment rank }\end{array}$ & $\begin{array}{l}\text { Symbolic } \\
\text { meaning }\end{array}$ & Assigned plant species & $\begin{array}{l}\text { Frequ } \\
\text {-ency }\end{array}$ \\
\hline 1 & Prosperity & $\begin{array}{l}\text { Camellia japonica L., Chrysanthemum morifolium Ram., Forsythia koreana Nakai, Kerria japonica } \\
\text { (L.) DC., Lilium tigrinum Ker-Gawl., Prunus mume Sieb. et Zucc., Rhododendron mucronulatum } \\
\text { Turcz., Rhododendron schlippenbachii Max., Rosa hybrida Hort. }\end{array}$ & 9 \\
\hline 2 & Cohesion & $\begin{array}{l}\text { Cymbidium goeringii (Reichb. fil.) Reichb. fil., Forsythia koreana Nakai, Magnolia kobus DC., } \\
\text { Magnoria parviflora Sieb. et Zucc., Punica granafum L., Pyrus serotina Rehder, Rhododendron } \\
\text { mucronulatum Turcz., Rhododendron schlippenbachii Max., Rhododendron weyrichii Maxim., } \\
\text { Rosa rugosa Thunb. }\end{array}$ & 10 \\
\hline 3 & Combination & $\begin{array}{l}\text { Camellia japonica L., Cosmos bipinnatus Cav., Forsythia koreana Nakai, Magnolia denudata } \\
\text { Desroux., Magnolia kobus DC., Rhododendron mucronulatum Turcz., Rhododendron } \\
\text { schlippenbachii Max., Rosa hybrida Hort., Rosa rugosa Thunb. }\end{array}$ & 9 \\
\hline 4 & Love & $\begin{array}{l}\text { Abeliophyllum distichum Nakai, Camellia japonica L., Chrysanthemum zawadskii Herbich var. } \\
\text { latilobum (Maxim.) Kitamura, Convallaria keiskei (Miq.) Miquel, Cornus officinalis Sieb. et Zucc., } \\
\text { Cosmos bipinnatus Cav., Forsythia koreana Nakai, Magnolia kobus DC., Prunus persica L., } \\
\text { Rhododendron mucronulatum Turcz., Rhododendron schlippenbachii Max., Rosa hybrida Hort. }\end{array}$ & 12 \\
\hline \multirow{3}{*}{5} & Mental & $\begin{array}{l}\text { Convallaria keiskei (Miq.) Miquel, Magnolia denudata Desroux., Magnolia kobus DC., } \\
\text { Rhododendron schlippenbachii Max., Zininia elegans Jacq. }\end{array}$ & 5 \\
\hline & Patience & $\begin{array}{l}\text { Chrysanthemum morifolium Ram., Cymbidium goeringii (Reichb. fil.) Reichb. fil., Forsythia } \\
\text { koreana Nakai, Rhododendron schlippenbachii Max., Rosa rugosa Thunb., Zininia elegans Jacq. }\end{array}$ & 6 \\
\hline & Vigor & $\begin{array}{l}\text { Camellia japonica L., Chrysanthemum morifolium Ram., Forsythia koreana Nakai, Rhododendron } \\
\text { schlippenbachii Max., Rhododendron yedoense Maxim. ex Regel var. poukhanense. Zininia elegans Jacq. }\end{array}$ & 6 \\
\hline \multirow{3}{*}{6} & Hope & Forsythia koreana Nakai, Pyrus serotina Rehder, Rhododendron mucronulatum Turcz. & 3 \\
\hline & Passion & $\begin{array}{l}\text { Camelliajaponica L., Rhododendron schlippenbachii Max., Rhododendron weyrichii Maxim., Rosa } \\
\text { hybrida Hort. }\end{array}$ & 4 \\
\hline & Richness & Chrysanthemum morifolium Ram., Nelumbo nucifera Gaertn. Rhododendron schlippenbachii Max. & 3 \\
\hline \multirow{3}{*}{7} & Development & $\begin{array}{l}\text { Forsythia koreana Nakai, Prunus mume Sieb. et Zucc., Prunus persica L., Rhododendron } \\
\text { schlippenbachii Max., Rhododendron weyrichii Maxim. } \\
\text { Rosa hybrida Hort., Zininia elegans Jacq. }\end{array}$ & 7 \\
\hline & Personality & $\begin{array}{l}\text { Chrysanthemum morifolium Ram., Cornus officinalis Sieb. et Zucc., Magnolia sieboldii K. Koch, } \\
\text { Prunus mume Sieb. et Zucc., Prunus persica L., Rhododendron mucronulatum Turcz., }\end{array}$ & 6 \\
\hline & Will & $\begin{array}{l}\text { Camellia japonica L., Chrysanthemum indicum L., Pulsatilla tongkangensis Y. N. Lee \& T. C. Lee, } \\
\text { Punica granafum L., Pyrus serotina Rehder, Zininia elegans Jacq. }\end{array}$ & 6 \\
\hline \multirow{3}{*}{8} & Chastity & $\begin{array}{l}\text { Camellia japonica L., Magnolia denudata Desroux., Magnolia kobus DC., Magnoria parviflora } \\
\text { Sieb. et Zucc., Rosa hybrida Hort., Rosa rugosa Thunb. }\end{array}$ & 6 \\
\hline & Local patriotism & $\begin{array}{l}\text { Camellia japonica L., Gardenia jasminoides Ellis var. grandiflora Nakai, Rhododendron } \\
\text { mucronulatum Turcz., Rhododendron schlippenbachii Max., Rosa hybrida Hort. }\end{array}$ & 5 \\
\hline & Strength & $\begin{array}{l}\text { Camellia japonica L., Helianthus annuus L., Prunus mume Sieb. et Zucc., Rhododendron } \\
\text { mucronulatum Turcz., Rhododendron schlippenbachii Max. }\end{array}$ & 5 \\
\hline \multirow{3}{*}{9} & Beauty & $\begin{array}{l}\text { Camellia japonica L., Narcissus spp., Pyrus serotina Rehder, Rhododendron schlippenbachii Max., } \\
\text { Rosa hybrida Hort. }\end{array}$ & 5 \\
\hline & Industry & $\begin{array}{l}\text { Camellia japonica L., Prunus armeniaca L. var. ansu Max., Rhododendron mucronulatum Turcz., } \\
\text { Rhododendron schlippenbachii Max. }\end{array}$ & 4 \\
\hline & Unsophisticated & $\begin{array}{l}\text { Chrysanthemum indicum L., Forsythia koreana Nakai, Magnolia sieboldii K. Koch, Magnoria parviflora } \\
\text { Sieb. et Zucc., Pyrus serotina Rehder, Rhododendron yedoense Maxim. ex Regel var. poukhanense }\end{array}$ & 6 \\
\hline \multirow{3}{*}{10} & Fidelity & Chrysanthemum morifolium Ram., Prunus mume Sieb. et Zucc., Rhododendron schlippenbachii Max. & 3 \\
\hline & Noble & Prunus mume Sieb. et Zucc. & 1 \\
\hline & Peace & $\begin{array}{l}\text { Convallaria keiskei (Miq.) Miquel, Forsythia koreana Nakai, Magnolia kobus DC., Prunus } \\
\text { armeniaca L. var. ansu Max. }\end{array}$ & 4 \\
\hline
\end{tabular}


governments in South Korea had designated floral emblems. Total 44 plant species were designated as floral emblems, and these did not include rose of Sharon, which is the national flower of South Korea. Most plant species used as floral emblems with high designation frequency were flowering trees or shrubs, and the ones with high frequency were Rhododendron spp., Rosa spp., Camellia spp., Magnolia spp., and Prunus spp. As a result of analyzing the symbolic meanings given to the plant species with top 10 designation frequency, it was found that each plant species had at minimum 11 to maximum 40 symbolic meanings, and the plant species with higher designation frequency tended to have more symbolic meanings. This result is due to the fact that, even if the same plant species is designated as the floral emblem, each local government used special, differentiated terms as the symbolic meaning. Many symbolic meanings of the floral emblems were historical/cultural sentiments or terms that reflected the values pursued by the local governments such as 'prosperity', 'richness', 'local patriotism', and 'citizenship' rather than the generally used floriography. Total 155 terms were used as symbolic meanings of floral emblems, with 'prosperity', 'cohesion', and combination' showing high frequency. Most plant species connected to the terms with top 10 frequency in symbolic meanings were the ones included in the top 10 floral emblem designation frequency.

The following summarizes the results of analyzing the symbolic meanings given to the plant species with high floral emblem designation frequency and the plant species assigned to the symbolic meanings that showed high frequency based on the two perspectives presented in the research methods. First, floral emblem designation frequency was high in plant species with high historical/cultural intimacy, and the symbolic meanings given to plant species showed high frequency in terms related to regional economic growth, cultivation of local community spirit, and personality required from local residents rather than floriography that is commonly perceived as the image of the plant itself. Moreover, various terms were connected to consciously reflect the goal or identity pursued by the local governments. As such, without sufficiently publicizing the diverse symbolic meanings given to the same plant species depending on the region, it is unlikely for the members of the community to perceive the specificity and distinction of the floral emblem and to give identity through the floral emblem. Thus, it is necessary to actively publicize the floral emblems to promote the awareness of the local residents. Second, floral emblems with low designation frequency tended to have relatively strong physical/emotional correlation with the local governments, and thus are expected to have high utilization in regional branding and tourism marketing. Therefore, it is necessary to establish a rational designation process and seek efficient utilization plans so that floral emblems can adequately reflect the regional characteristics and values pursued by the local governments.

This study can be used as the basic data when the local governments host festivals or exhibitions in flower themes for garden tourism, in the design industry such as symbols, icons and sculptures reflecting the symbolic meanings of the floral emblems, or when the floral emblems are newly designated with change in the administrative division. Since the scope of this research is limited to the investigation and analysis of the current state of floral emblem designation by the local governments as well as symbolic meanings, there were limitations in that more concrete research could not be conducted on the perception of the members of the community about the floral emblems, or their social and cultural use. It is necessary to conduct further studies on use of floral emblems and perception of local residents in tourism and the design industry.

\section{References}

Bang, K.J. and J.S. Lee. 1995. Studies on planting distribution status of landscaping plants in Korea. J. Korean Inst. Landsc. Archit. 23(1):67-94.

Benfield, R.W. 2013. Garden tourism. Boston, MA: CAB International.

Bird, A.J., S. Goldsberry, and J.P.K. Bird. 1987. Hawaiian flower lei making. Honolulu, HI: University of Hawaii Press.

Chang, S.Y. 2016. Design development for fashion cultural product using traditional flower patterns: Focused on peony pattern. J. Korean Soc. Fashion Des. 16(2):159-171. https://doi.org/10.18652/2016.16.2.10 
Chu, J.S., I. Kim, and K.Y. Huh. 2018. Recognition assessment and structural equation model of participants after learning floral design courses as vocational education. J. People Plants Environ. 21(4):279-292. https://doi.org/10.11628/ksppe.2018.21.4.279

Connell, J. 2004. The purest of human pleasures: The characteristics and motivations of garden visitors in Great Britain. Tour. Manag. 25(2):229-247. https://doi.org /10.1016/j.tourman.2003.09.021

Criley, R.A. 1992. Hawaii's lei flower industry. HortScience 27(6): 490-492. https://doi.org/10.21273/HORTSCI.27. 6.490

Goss, J. 1993. The 'magic of mall': An analysis of form, function, and meaning in the contemporary retail. Ann. Am. Assoc. Geogr. 83(1):18-47.

Hong, J.H. and H.K. Kim. 2011. The development of woman's daily Hanbok textile design appling Korea traditional flower pattern. J. Korean Soc. Cloth. Ind. 13(6):848-855. https://doi.org/10.5805/KSCI.2011.13.6.848

Jeongseon. 2019. Traveling to Jeongseon: Donggang pasqueflower festival. Retrieved from http://jeongseon. go.kr/tour/jeongseontour/festivals

Jung, J.S. 2004. The development of textile design and the manufacture of cultural merchandise by using Lotus flower patterns( II ). J. Korean Soc. Cloth. Ind. 6(4):421-426.

Kim, I. and K.Y. Huh. 2019. Enhancement of recognition on strategic cooperation between Vietnam and Korea in transition from urban tourism to green tourism.
International conference proceedings of Vietnam-Korea strategic cooperative partnership 2009-2019(pp. 73-89). Ho Chi Minh, Vietnam.

Kim, S.Y. 2009. Development of fashion culture goods designs motivated by ume flowers. Res. J. Costume Cult. 17(6):972-980.

Korea Forest Service. 2007. The rational improvement plan for the application, management, production, and distribution of landscape trees. Daejeon, Korea.

Korean Statistical Information Service(KOSIS). 2019. Designated (Legal) Tourist Attractions and Visitors. Retrieved from http://kosis.kr/statHtml/statHtml.do?orgI $\mathrm{d}=$ 733\&tblId=DT_733001_J008

Ministry of the Interior and Safety. 2019. The national flower - Mugunghwa. Retrieved from https://www.mois.go.kr/e ng/sub/a03/nationalSymbol_3/screen.do

Rho, J.H. 1998. A study on the designation characteristics of symbolic plants in province of self-government. J. Korean Inst. Tradit. Landsc. Archit. 16(3):81-93.

Song, H.R. 2010. A study on fashion design by application of Korean traditional flowered-wall motives: focused on flower patterns. J. Korean Soc. Fashion Des. 10(1):41-56.

Wyse Jackson, P.S. and L.A. Sutherland. 2000. International agenda for botanic gardens in conservation (pp. 12-14). Surrey, England: Botanic Gardens Conservation International.

Ziegler, C. 2007. Favored flowers: Culture and economy in a global system. Durham, NC: Duke University Press. 